\title{
1991-1992 Editorial consultants (continued)
}

\section{advertisers' index}

T

Takacs, Steven Martin, Newbury, Ohio

Taylor, Jerry A., Southfield, Mich

Todd, Carson, Tulsa, Okla

\section{V}

Van Buskirk, Richard L., Sarasota, Fla

Venditto, Michael A., Langhorne, Pa W

Ward, Robert C., East Lansing, Mich Wenig, Paul E., Troy, Mich

Wickless, Larry A., Farmington Hills, Mich

Williams, Basil B. Jr, Ghent, NY

Wolf, David L., Trenton, Mich

\section{$\mathbf{Z}$}

Zuzga, John J., Orland Park, Ill

\section{Curatek Pharmaceuticals}

MetroGel, 1477-1482

Deborah Heart \& Lung Center, 1490

Flint Osteopathic Hospital, 1522

Forest Laboratories, Inc.

Levothroid, 1447, 1448

Eli Lilly and Company

Lorabid, 1465-1468

McNeil Consumer Products

Pediaprofen, 1471, 1472, 1488

Tylenol, 1445

\section{National Osteopathic}

Foundation, 1525

Ohio University College of

Osteopathic Medicine, 1500

Pfizer Labs Division

Norvasc, 1491-1496

Procardia XL, 1473, 1474

Zithromax, 1533-1538

\section{Rhone-Poulenc Rorer}

Pharmaceuticals

Dilacor, 1458-1460

Roche Products, Inc.

Valium, 1485
G. D. Searle and Company Maxaquin, 1513-1517

\section{SmithKline Beecham}

Augmentin, Cover 4

\section{Sterling Health}

Bayer Select, 1462, 1463

3M Pharmaceuticals

Autohaler, Cover 3

Upjohn

Vantin, 1449-1456

Xanax, Cover 2-1442 